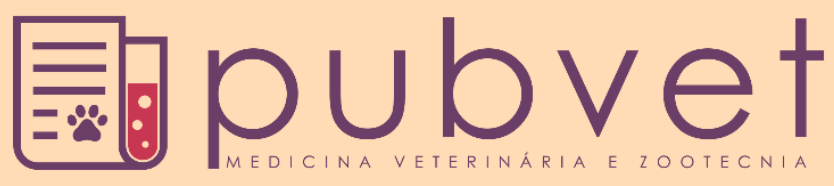

https://doi.org/10.31533/pubvet.v15n02a753.1-7

\title{
Ingestão acidental de ibuprofeno por cão filhote: Relato de caso
}

\author{
Leonardo Bianchi de Oliveira ${ }^{1 * 9}$, Anna Victoria Antunes ${ }^{19}$, Simone Tostes de Oliveira ${ }^{2} \mathbb{9}$, \\ Thaís Ribeiro Penna Paiva ${ }^{1} \mathbb{9}$, Tilde Rodrigues Froes ${ }^{2} \mathbb{9}$, Marlos Gonçalves Sousa ${ }^{2} \mathbb{9}$ \\ ${ }^{1}$ Médico(a) Veterinário(a) Residente de Clínica Médica de Pequenos Animais do Hospital Veterinário da Universidade Federal do Paraná. \\ Curitiba-PR. Brasil \\ ${ }^{2}$ Professor (a) da Universidade Federal do Paraná, Departamento Medicina Veterinária. Curitiba - PR. Brasil \\ *Autor para correspondência, E-mail: oliveiraleo1@gmail.com
}

\begin{abstract}
Resumo. O ibuprofeno é amplamente utilizado na medicina devido suas características antipiréticas, anti-inflamatórias e analgésicas, e é relativamente seguro para humanos, mas quando administrados, de forma indevida, para animais de companhia, possuem efeitos colaterais indesejáveis. Os relatos da ingestão acidental de ibuprofeno por cães mostram que sinais gastrointestinais e renais de intoxicação são os mais comuns. $\mathrm{O}$ tratamento envolve a prevenção e manejo dos danos instalados. O objetivo do trabalho foi relatar o caso de uma cadela filhote que ingeriu uma cartela de ibuprofeno e manifestou alterações gastrointestinais e renais, e houve sucesso na terapêutica empregada.
\end{abstract}

Palavras chave: AINEs, intoxicação, úlcera gástrica

\section{Accidental ingestion of ibuprofen by puppy dog: Case report}

\begin{abstract}
Ibuprofen is widely used in medicine due to its antipyretic, anti-inflammatory, and analgesic characteristics. It is relatively safe for humans, but when improperly administered to pets, it has undesirable side effects. Reports of accidental ingestion of ibuprofen by dogs show that gastrointestinal and renal signs of intoxication are the most common. Treatment involves preventing and managing installed damage. The main of this case report was present a puppy dog that ingested an ibuprofen packet and manifested gastrointestinal and renal alterations, and the therapy employed was successful.
\end{abstract}

Keywords: NSAIDs, intoxication, gastric ulcer

\section{Ingestión accidental de ibuprofeno por un cachorro: Reporte de un caso}

Resumen. El ibuprofeno se usa ampliamente en medicina debido a sus características antipiréticas, antiinflamatorias y analgésicas, y es relativamente seguro para los humanos, pero cuando se administra incorrectamente a las mascotas, tiene efectos secundarios indeseables. Los informes de ingestión accidental de ibuprofeno por perros muestran que los signos de intoxicación gastrointestinal y renal son los más comunes. El tratamiento implica prevenir y gestionar los daños instalados. El objetivo del trabajo fue reportar el caso de una cachorra que ingirió un paquete de ibuprofeno y manifestó alteraciones gastrointestinales y renales, y la terapia empleada fue exitosa.

Palabras clave: AINEs, intoxicación, úlcera gástrica

\section{Introdução}

O ibuprofeno é um anti-inflamatório não esteroidal (AINEs), comumente utilizado em seres humanos para tratar dor, febre e inflamação, e embora seja um fármaco relativamente seguro nessa espécie, a administração indevida ou acidental pode ser prejudicial para os cães. Na medicina veterinária, o 
ibuprofeno tem uma estreita margem de segurança terapêutica, podendo causar toxidade em vários órgãos (Fitzgerald et al., 2006). Os AINEs inibem a enzima ciclooxigenase (COX) que permite a síntese de prostaglandinas (PGs) que mediam a inflamação e a febre (Khan \& McLean, 2012). Existem duas isoenzimas da COX, a COX-1 e a COX-2. A COX-1 parece estar presente naturalmente no corpo, e está envolvida em importantes funções fisiológicas, além de ser encontrada principalmente no rim, estômago, endotélio e plaquetas. Sua inibição, como é o caso da ação do ibuprofeno, pode levar a efeitos adversos associados à AINEs, como ulceração gastrointestinal e lesão renal (Bennett et al., 1996).

A toxidade dos AINES em cães é reforçada devido a circulação entero-hepática, que permite que a droga seja excretada pela bile para ser reabsorvida pelo intestino delgado, resultando em reexposição, e tornando-se um fator crítico para indução de lesão, principalmente em intestino delgado (Curry et al., 2005). A lesão renal, irritação e hemorragias gastrointestinais são os efeitos mais comuns relatados na ingestão de doses tóxicas de ibuprofeno em cães (McEvoy, 2000). Os cães tratados com ibuprofeno por via oral $8 \mathrm{mg} / \mathrm{kg} / \mathrm{dia}$ ou $16 \mathrm{mg} / \mathrm{kg} /$ dia por 30 dias desenvolveram ulcerações gástricas e sinais clínicos relacionados a distúrbios gastrointestinais (Adams et al., 1969). A dose de ibuprofeno considerada letal para cães é acima de $600 \mathrm{mg} / \mathrm{kg}$ (Dunayer, 2004).

Em cães clinicamente normais (aqueles que não apresentam sinas neurológicos ou gástricos) a indução de emêse, especialmente dentro de duas horas da ingestão, pode ser uma alternativa para minimizar a absorção do ibuprofeno (Dunayer, 2004). O tratamento da intoxicação consiste no controle dos sinais clínicos e terapia de suporte por meio de fluidoterapia, antieméticos, analgésicos, protetores de mucosas, inibidor da bomba de prótons, antagonista de receptor $\mathrm{H} 2$ e análogo da prostaglandina (Bolfer et al., 2014).

O presente estudo objetivou relatar as alterações decorrentes da ingestão acidental de ibuprofeno por um cão filhote, bem como o sucesso da terapia utilizada.

\section{Material e métodos}

Foi atendida uma cadela da raça Buldogue Francês de cinco meses de idade, não castrada e pesando $7,2 \mathrm{~kg}$. Na anamnese, tutor relatou que a cadela mastigou uma cartela de ibuprofeno $600 \mathrm{mg}$ duas horas antes da consulta, porém não soube dizer a quantidade exata que animal pudesse ter ingerido, além do mais, relatou que antes do ocorrido, o animal estava hígido, e negou qualquer administração medicamentosa intencional. No exame físico, a única alteração observada foi a presença de dor à palpação abdominal na região epigástrica.

Diante do histórico clínico, foi optado pela internação do animal. Foram coletadas amostras de sangue para realização de exames complementares para acompanhamento da paciente durante sua permanência no internamento. Após o segundo dia de internação houve aumento da creatinina e ureia sérica $(2,7 \mathrm{mg} / \mathrm{dL}$ e $166,8 \mathrm{mg} / \mathrm{dL}$, respectivamente), mas os níveis séricos retornaram para a faixa de normalidade no terceiro dia de internação. Com relação a série vermelha (eritrócitos, hematócrito e hemoglobina) houve diminuição gradativa ao longo dos dias, até a paciente desenvolver anemia normocítica e normocrômica leve. Não houve alterações nas enzimas hepáticas (FA e ALT). Após um dia de internação foi coletada amostra de urina para urinálise, sendo observado duas cruzes de proteínas, uma cruz de cilindros hialinos, e relação proteína: creatinina urinária de 3 , sendo que $\geq$ de 0,5 indica aumento por processo patológico.

A ultrassonografia abdominal evidenciou alterações gástricas e renais. O estômago apresentou-se parcialmente contraído, preenchido por conteúdo gasoso/mucoso e com um espessamento focal $(0,6 \mathrm{~cm})$ na junção piloroduodenal (Figura 1A), indicando um processo inflamatório gástrico focal, e adjacente à transição piloroduodenal notou-se defeitos na mucosa, delineados por microbolhas acumuladas no local da erosão (Figura 1B), tendo como principal diagnóstico diferencial uma pequena úlcera gástrica de aproximadamente $0,2 \mathrm{~cm}$.

O rim apresentava aumento discreto no seu tamanho e aumento difuso da ecogenicidade do córtex renal (Figura 2), podendo indicar nefropatia aguda, em consonância com histórico clínico da paciente.

A paciente foi encaminhada para a internação (Figura 3A) para dar início ao tratamento por meio da administração intravenosa de solução de ringer com lactato na taxa de $60 \mathrm{~mL} / \mathrm{kg} / 24$ horas, omeprazol 
$1 \mathrm{mg} / \mathrm{kg} / \mathrm{IV}$ SID, butilbrometo de escopolamina + dipirona sódica $25 \mathrm{mg} / \mathrm{kg} / \mathrm{IV}$ BID e ondansetrona 0,5 $\mathrm{mg} / \mathrm{kg} / \mathrm{IV}$ TID, e ao longo do dia os parâmetros vitais foram acompanhados e se mantiveram estáveis. Após o resultado da ultrassonografia, o sucralfato $30 \mathrm{mg} / \mathrm{kg} / \mathrm{VO}$ TID foi adicionado à prescrição. No segundo dia de internamento, foi acrescida metadona $0,2 \mathrm{mg} / \mathrm{kg} / \mathrm{IM}$ BID. O débito urinário se manteve entre 2,7 e $5,2 \mathrm{~mL} / \mathrm{kg} / \mathrm{h}$.

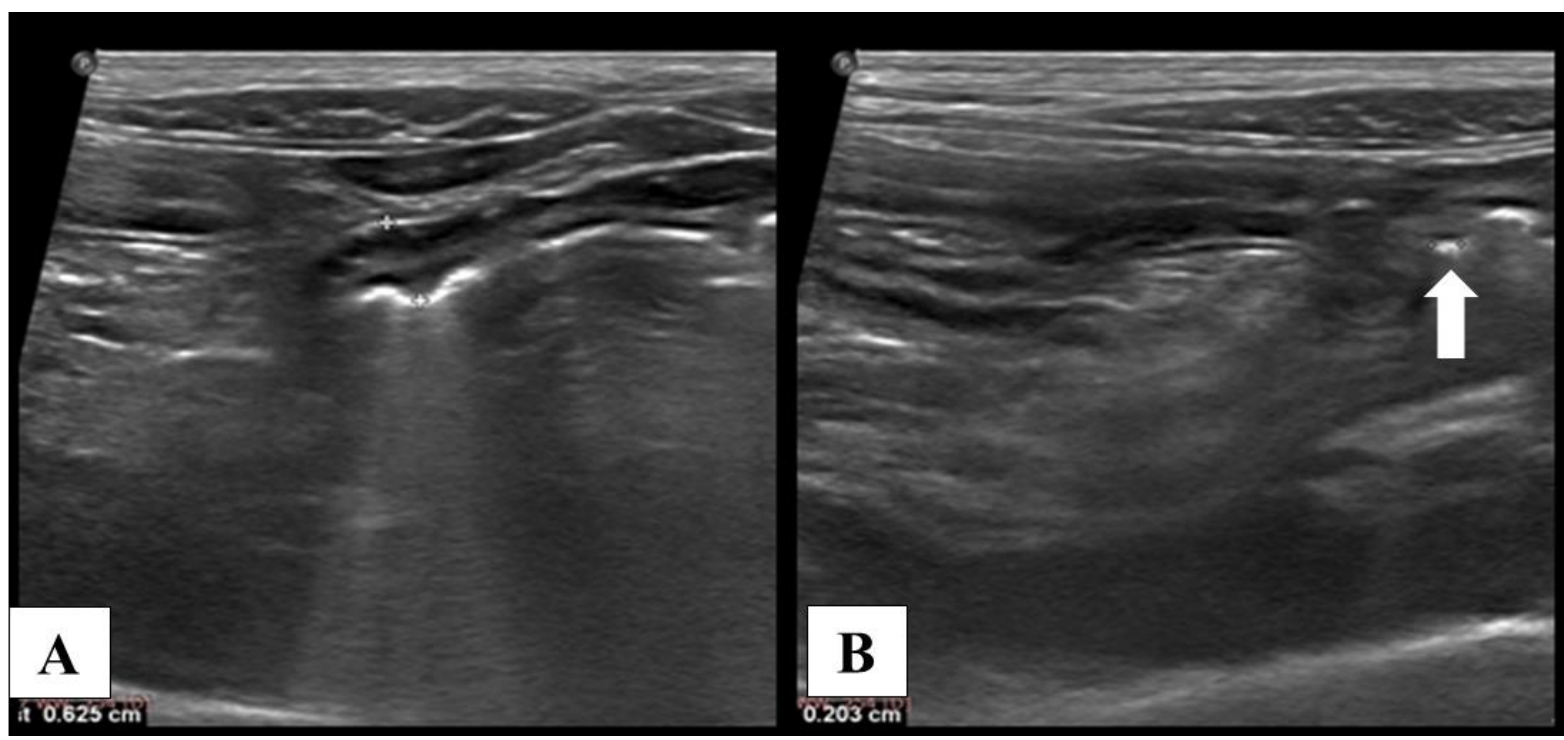

Figura 1. Ultrassonografia abdominal de cadela intoxicada por ibuprofeno. (A): eixo longitudinal do estômago, sendo evidenciado leve espessamento da parede de $0,6 \mathrm{~cm} ;(\mathbf{B})$ : eixo longitudinal do estômago, sendo evidenciado uma área focal hiperecóica, aparentemente uma erosão de $0,2 \mathrm{~cm}$ (seta), em transição piloroduodenal e subjetiva redução da espessura da camada mucosa.

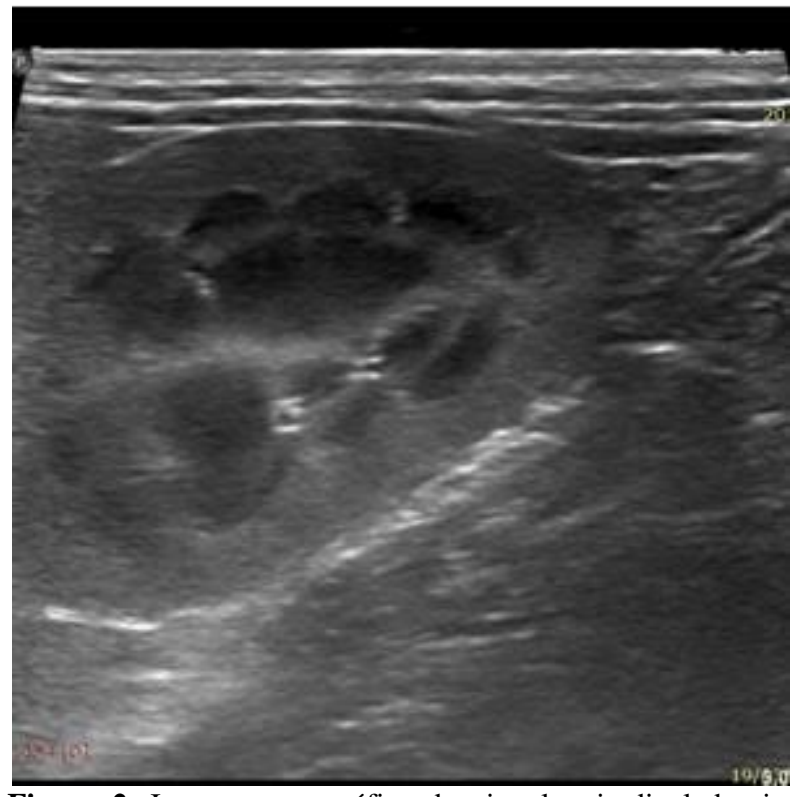

Figura 2. Imagem sonográfica do eixo longitudinal do rim direito, sendo evidenciado o aumento da ecogenicidade da cortical, sugerindo nefropatia aguda.

Além dos parâmetros vitais e exames complementares, foi realizado o acompanhamento da defecação, micção, êmese e ingestão alimentar da paciente, que se alimentou espontaneamente no segundo dia. No terceiro dia de internação apresentou episódios de hematêmese (Figura 3B) e melena, mas que foram cessados nos dias subsequentes. No decorrer dos sete dias de internação, os parâmetros se mantiveram dentro da normalidade e a paciente apresentou-se hidratada. 
Após sete dias, a cadela recebeu alta e para casa foi prescrito omeprazol $10 \mathrm{mg}(0,7 \mathrm{mg} / \mathrm{kg} / \mathrm{VO} \mathrm{SID}$ durante 7 dias), ondansetrona $4 \mathrm{mg}(0,5 \mathrm{mg} / \mathrm{kg} / \mathrm{VO} \mathrm{BID}$, durante 4 dias), sucralfato $2 \mathrm{~g} / 10 \mathrm{~mL}$ (30 $\mathrm{mg} / \mathrm{kg} / \mathrm{VO}$ TID durante 10 dias) e butilbrometo de escopolamina $10 \mathrm{mg}(0,2 \mathrm{mg} / \mathrm{kg} / \mathrm{BID}$ VO por 5 dias $)$. Após uma semana, o proprietário foi contatado e o mesmo informou que a paciente estava bem e não apresentava qualquer alteração dos sinais clínicos. Após cinco meses a paciente retornou, e foram coletados exames de hemograma e bioquímico para avaliação hepática e renal, estando todos dentro da normalidade.

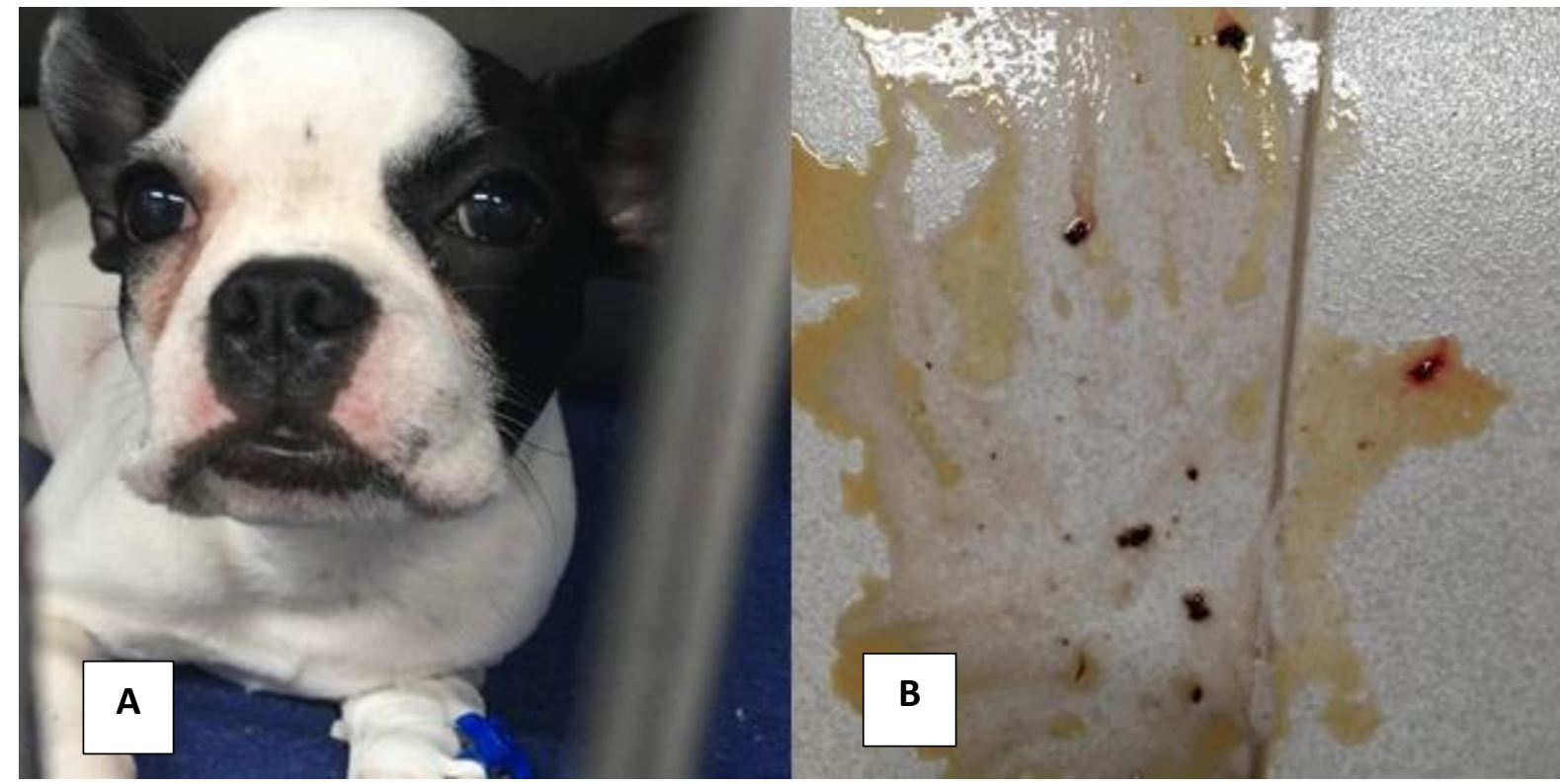

Figura 3. (A): Imagem fotográfica da cadela filhote, na internação; (B): Presença de pequenos coágulos no conteúdo estomacal eliminado.

\section{Resultados e discussão}

No passado, para os cães era recomendado uma dose de $5 \mathrm{mg} / \mathrm{kg}$ de ibuprofeno para tratamento, desejando efeitos anti-inflamatório e analgésicos (Kore, 1990), porém uma dose próxima a dose terapêutica pode causar úlceras gástricas e perfurações nessa espécie (Ramprabhu et al., 2001; Tams, 2003). Dessa forma, o ibuprofeno não é mais recomendado para cães devido a sua estreita margem de segurança, sendo substituído por outros anti-inflamatórios mais seguros para a espécie (Bates, 2016; Cathers et al., 2000; Perry, 1998).

Durante a anamnese não foi possível determinar a quantidade exata de ibuprofeno que o animal pudesse ter ingerido ou quantos comprimidos havia na cartela, porém era sabido que cada comprimido possuía $600 \mathrm{mg}$ de ibuprofeno, e apenas a ingestão de um único comprimido daria uma dose aproximada de $84 \mathrm{mg} / \mathrm{kg}$. O ibuprofeno em doses superiores a $175 \mathrm{mg} / \mathrm{kg}$ pode levar a lesão renal aguda por isquemia em cães, pois as prostaglandinas são responsáveis por vasodilatação e manutenção do fluxo renal (Villar et al., 1998). Doses superiores a $400 \mathrm{mg} / \mathrm{kg}$ em cães produziram efeitos no sistema nervoso central, como depressão, convulsão e coma (Villar et al., 1998). Nesse caso não houve alterações neurológicas.

Um estudo demonstrou que uma única dose de ingestão experimental de $125 \mathrm{mg} / \mathrm{kg}$ foi associada a sinais gastrointestinais, como gastrite, êmese, melena, erosões no antro pilórico e no piloro (Adams et al., 1969), alterações também encontradas no paciente do presente trabalho. Essas alterações gastrointestinais estão relacionadas com a inibição da COX1 e consequentemente inibição da síntese de prostaglandina, um sinalizador químico celular responsável pela proteção gástrica (Holland \& Chastain, 1995). A inibição de prostaglandinas leva a uma redução do fluxo sanguíneo na mucosa e diminuição na produção de muco, sendo o antro pilórico e o piloro as regiões mais acometidas (Sullivan \& Yool, 1998), como encontrado nesse caso, no qual tal alteração foi evidenciada no ultrassom abdominal. O ibuprofeno é um inibidor irreversível não especifico de COX, ou seja, inibe COX1 e COX2 na mesma proporção (Dunayer, 2004). Na terapia com AINEs, os inibidores seletivos de COX2 são preferencialmente escolhidos, pois inibem a formação de prostaglandinas associados a inflamação, mas poupam as prostaglandinas produzidas pela COX1 envolvidas na homeostase (Curry et al., 2005). 
$\mathrm{Na}$ consulta inicial do presente caso, o tutor não relatou alteração no quadro clínico da paciente, possivelmente devido a urgência em que foi trazida para o hospital. Durante a internação foi observada inicialmente inapetência, e depois vômito no terceiro dia de internação, sinais compatíveis com a intoxicação por ibuprofeno, com os efeitos de toxinas urêmicas sobre a zona de disparo dos quimiorreceptores do centro bulbar da êmese, redução na excreção de gastrina, resultando em aumento de secreção ácida gástrica e irritação do trato gastrointestinal secundário a vasculite urêmica (Seabra, 2015).

O ibuprofeno possui metabolização hepática e excreção renal, e há evidências que sofre recirculação entero-hepática, assim como outros AINEs (Richardson, 2000). Pela análise das enzimas ALT e FA da paciente, não houve indícios que a ingestão do ibuprofeno causou danos hepático.

Além de lesão renal por alteração do fluxo sanguíneo, os AINEs podem causar nefrite intersticial, hematúria, necrose papilar renal, glomerulite, glomerulonefrite, hipercalemia e síndrome nefrótica (Riboldi et al., 2012). A paciente apresentou aumento da azotemia de forma abrupta no segundo dia de internação, possivelmente por vasoconstrição arteriolar aferente causando diminuição na taxa de filtração glomerular e consequentemente insuficiência renal aguda (Nelson \& Couto, 2015). O histórico clínico da paciente, associado as alterações renais evidenciadas na ultrassonografia (aumento discreto no seu tamanho e aumento na ecogenicidade do córtex renal), corrobora com suspeita de lesão renal aguda (Thrall, 2019). A doença renal pré-existente, hipovolemia (seja por desidratação concomitante) e hipotensão podem aumentar o risco de toxidade renal do ibuprofeno (Kore, 1990), porém, no presente estudo, a paciente era jovem, sem histórico mórbido, permaneceu clinicamente hidratada durante a internação e sua pressão sistólica avaliada permaneceu dentro dos valores de referência para a espécie.

A proteinúria severa, diagnosticada por meio da relação proteína-creatinina urinária (RPC), associada a sedimento inativo é a alteração mais comum da glomerulopatia, e comumente são encontrados cilindros hialinos nas doenças renais associados a glomerulopatias (Nelson \& Couto, 2015). Todas essas alterações foram encontradas, o que reafirma que o AINE pode levar a toxidade renal. A avaliação precisa da proteinúria envolve três elementos chaves: persistência, localização e magnitude da proteinúria (Lees et al., 2005) e a proteinúria persistente é definida como proteinúria detectada em três ou mais ocasiões, com diferença de duas semanas ou mais (Vaden \& Elliott, 2016). No presente trabalho, a urinálise com RPC foi realizada apenas uma vez, porém o ideal seria se tivesse havido repetições, o que auxiliaria no diagnóstico e no prognóstico do paciente (Stolf, 2011). Segundo Lees et al. (2005), proteinúria persistente maior que 2 sugere glomerulopatia.

A estimativa da taxa de filtração glomerular (TGF) é o padrão ouro para avaliação de função renal, e a avaliação da creatinina sérica e dimetilginina simétrica (SDMA) são os principais meios para avaliar função renal em cães na prática clínica (Polzin, 2013; Nabity et al., 2015). No presente relato foi observada azotemia nos exames da paciente no dia seguinte a sua internação, com aumento de creatinina e uréia. Caso tivesse sido mensurado a SMDA, esta estaria aumentada também, uma vez que é ainda mais sensível que a creatinina.

A paciente apresentou decréscimo gradual dos eritrócitos, hematócrito e hemoglobina até desenvolver anemia normocítica e normocrômica leve, essa alteração possivelmente deve-se a perda sanguínea no local da úlcera estomacal, alteração também encontrada por Ramprabhu et al. (2001), e nesse estudo a perda sanguínea foi evidenciada através da endoscopia digestiva e também pela presença de melena.

O tratamento da intoxicação por AINEs visa prevenir ou tratar doenças gastrointestinais e danos renais (Bates, 2016). A fluidoterapia é essencial para facilitar a perfusão de órgãos alvos, e a desidratação deve ser rigorosamente monitorada para diminuir o risco de lesão renal (Fitzgerald et al., 2006). Durante a internação, pela avaliação de tempo de preenchimento capilar, turgor cutâneo, presença ou não de enoftalmia e avaliação da consciência, foi possível avaliar de forma subjetiva a hidratação da paciente, e a mesma permaneceu corretamente hidratada.

Há evidências que cães com úlceras induzidas por AINEs, o tratamento ideal envolve famotidina, omeprazol e misoprostol (Osweiler \& Carson, 1997). O sucralfato é recomendado apenas se houver suspeita ou confirmação de úlcera gástrica, e deve ser administrado duas horas antes do bloqueador de receptores de histamina ou bloqueador da bomba de prótons, pois exige um ambiente ácido para melhor 
eficácia (Bates, 2016). A paciente recebeu inicialmente omeprazol, butilbrometo de escopolamina + dipirona sódica e ondansetrona; após a confirmação de uma úlcera gástrica pela ultrassonografia abdominal, o sucralfato foi adicionado à prescrição. No segundo dia foi adicionada metadona para auxiliar na analgesia, pois além do desconforto abdominal a palpação, a paciente apresentava apetite seletivo, e deduziu-se que pudesse ser devido a dor.

O prognóstico é bom em animais que recebem o tratamento imediato logo após a ingestão; animais que ingerem uma superdosagem com início agudo dos sinais neurológicos, perfurações gastrointestinais ou exposição crônica tem um prognostico reservado (Bates, 2016).

\section{Conclusão}

Nesse caso relatado, a paciente foi trazida para consulta de forma imediata, antes de apresentar os sinais clínicos associados a intoxicação por AINEs, dessa forma, foi possível instituir o tratamento precocemente, o que beneficiou a recuperação e melhorou o prognóstico.

\section{Referências}

Adams, S. S., Bough, R. G., Cliffe, E. E., Lessel, B., \& Mills, R. F. N. (1969). Absorption, distribution and toxicity of ibuprofen. Toxicology and Applied Pharmacology, 15(2), 310-330. https://doi.org/10.1016/0041-008X(69)90032-5

Bates, N. (2016). Ibuprofen toxicosis. Companion Animal, 21(6),346-350. https://doi.org/10.12968/coan.2016.21.6.346

Bennett, W. M., Henrich, W. L., \& Stoff, J. S. (1996). The renal effects of nonsteroidal antiinflammatory drugs: Summary and recommendations. American Journal of Kidney Diseases, 28(1), S56-S62. https://doi.org/10.1016/S0272-6386(96)90570-3

Bolfer, L., McMichael, M., Ngwenyama, T. R., \& O'Brien, M. A. (2014). Treatment of Ibuprofen Toxicosis in a Dog with IV Lipid Emulsion. Journal of the American Animal Hospital Association, 50(2), 136-140. https://doi.org/10.5326/JAAHA-MS-5979

Cathers, T. E., Isaza, R., \& Oehme, F. (2000). Acute ibuprofen toxicosis in a ferret. Journal of the American Veterinary Medical Association, 216(9), 1426-1428. https://doi.org/10.2460/javma.2000.216.1426

Curry, S. L., Cogar, S. M., \& Cook, J. L. (2005). Nonsteroidal Antiinflammatory Drugs: A Review. Journal of the American Animal Hospital Association, 41(5), 298-309. https://doi.org/10.5326/0410298

Dunayer, E. (2004). Ibuprofen toxicosis in dogs, cats, and ferrets. Veterinary Medicine-Bonner Springs Then Edwardsville, 99, 580-586.

Fitzgerald, K. T., Bronstein, A. C., \& Flood, A. A. (2006). "Over-The-Counter" Drug Toxicities in Companion Animals. Clinical Techniques in Small Animal Practice, 21(4), 215-226. https://doi.org/10.1053/j.ctsap.2006.10.006

Holland, M., \& Chastain, C. B. (1995). Uses and misuses of aspirin. Kirk's Current Veterinary Therapy XII, Small Animal Practice. Philadelphia, PA, WB Saunders.

Khan, S. A., \& McLean, M. K. (2012). Toxicology of Frequently Encountered Nonsteroidal AntiInflammatory Drugs in Dogs and Cats. Veterinary Clinics of North America: Small Animal Practice, 42(2), 289-306. https://doi.org/10.1016/j.cvsm.2012.01.003

Kore, A. M. (1990). Toxicology of Nonsteroidal Antiinflammatory Drugs. Veterinary Clinics of North America: Small Animal Practice, 20(2), 419-430. https://doi.org/10.1016/S0195-5616(90)50036-4

Lees, G. E., Brown, S. A., Elliott, J., Grauer, G. F., \& Vaden, S. L. (2005). Assessment and Management of Proteinuria in Dogs and Cats: 2004 ACVIM Forum Consensus Statement (Small Animal). Journal of Veterinary Internal Medicine, 19(3), 377-385. https://doi.org/10.1111/j.1939-1676.2005.tb02713.x

McEvoy, G. K. (2000). Ibuprofen. In American hospital formulary service drug information (Bethesda (p. 1815). Pharmaceutical Press.

Nelson, R., \& Couto, C. G. (2015). Medicina interna de pequenos animais. Elsevier Brasil.

Osweiler, G., \& Carson, T. L. (1997). Household drugs. In The Handbook of Small Animal Practice, $3 r d$.

Perry, H. (1998). Acetaminophen. In Clinical Management of Poisoning and Overdose. 
Ramprabhu, R. A., Prathaban, S., Nambi, A. P., Nagarajan, B., \& Dhanapalan, P. (2001). Endoscopic evaluation of gastric mucosa after oral administration of ibuprofen in dogs. Veterinarski Arhiv, 71(1), 47-52.

Riboldi, E., Lima, D. A., \& Dallegrave, E. (2012). Sensibilidade espécie-específica aos antiinflamatórios não esteroidais: humanos X animais de companhia. Arquivo Brasileiro de Medicina Veterinária e Zootecnia, 64(1), 39-44. https://doi.org/10.1590/S0102-09352012000100006

Richardson, J. A. (2000). Management of Acetaminophen and Ibuprofen Toxicoses in Dogs and Cats. Journal of Veterinary Emergency and Critical Care, 10(4), 285-291. https://doi.org/10.1111/j.14764431.2000.tb00013.x

Seabra, C. I. R. (2015). Farmacocinética do ibuprofeno. http://hdl.handle.net/10284/5305

Stolf, L. C. (2011). Clínica de Pequenos Animais. Veterinarian Docs, 42-45.

Sullivan, M., \& Yool, D. A. (1998). Gastric disease in the dog and cat. The Veterinary Journal, 156(2), 91-106. https://doi.org/10.1016/S1090-0233(05)80035-8

Tams, T. R. (2003). Handbook of Small Animal Gastroenterology. Saunders. https://books.google.com.br/books?id=2rRNYfOIoaEC

Thrall, D. E. (2019). Diagnóstico de radiologia veterinária. Elsevier Editora Ltda.

Vaden, S. L., \& Elliott, J. (2016). Management of Proteinuria in Dogs and Cats with Chronic Kidney Disease. Veterinary Clinics of North America: Small Animal Practice, 46(6), 1115-1130. https://doi.org/10.1016/j.cvsm.2016.06.009

Villar, D., Buck, W. B., \& Gonzalez, J. M. (1998). Ibuprofen, aspirin and acetaminophen toxicosis and treatment in dogs and cats. Veterinary and Human Toxicology, 40(3), 156-162. http://europepmc.org/abstract/MED/9610496

\section{Histórico do artigo:}

Recebido: 18 de setembro de 2020

Aprovado: 17 de outubro de 2020.

Licenciamento: Este artigo é publicado na modalidade Acesso Aberto sob a licença Creative Commons Atribuição 4.0 (CC-BY 4.0), a qual permite uso irrestrito, distribuição, reprodução em qualquer meio, Disponível online: 22 de dezembro de 2020. desde que o autor e a fonte sejam devidamente creditados 